\title{
Understanding the effects of a polymer on the surface dissolution of pharmaceutical cocrystals using combined experimental and molecular dynamics simulation approaches
}

Preyanthiny Kirubakaran, Ke Wang, lan Rosbottom, Richard Barrie Michael Cross, and Mingzhong Li

Mol. Pharmaceutics, Just Accepted Manuscript • DOI: 10.1021/

acs.molpharmaceut.9b00955 - Publication Date (Web): 30 Dec 2019

Downloaded from pubs.acs.org on December 31, 2019

\section{Just Accepted}

"Just Accepted" manuscripts have been peer-reviewed and accepted for publication. They are posted online prior to technical editing, formatting for publication and author proofing. The American Chemical Society provides "Just Accepted" as a service to the research community to expedite the dissemination of scientific material as soon as possible after acceptance. "Just Accepted" manuscripts appear in full in PDF format accompanied by an HTML abstract. "Just Accepted" manuscripts have been fully peer reviewed, but should not be considered the official version of record. They are citable by the Digital Object Identifier (DOIß). "Just Accepted" is an optional service offered to authors. Therefore, the "Just Accepted" Web site may not include all articles that will be published in the journal. After a manuscript is technically edited and formatted, it will be removed from the "Just Accepted" Web site and published as an ASAP article. Note that technical editing may introduce minor changes to the manuscript text and/or graphics which could affect content, and all legal disclaimers and ethical guidelines that apply to the journal pertain. ACS cannot be held responsible for errors or consequences arising from the use of information contained in these "Just Accepted" manuscripts. 


\title{
Understanding the effects of a polymer on the surface dissolution of pharmaceutical cocrystals using combined experimental and molecular dynamics simulation approaches
}

\author{
Preyanthiny Kirubakarant, Ke Wangt, lan Rosbottom, Richard Barrie Michael \\ Crosst‡ and Mingzhong Li,t
}

\begin{abstract}
†School of Pharmacy, De Montfort University, The Gateway, Leicester, LE1 9BH, UK ‡Department of Chemical Engineering, Imperial College London, London, SW7 2BX, UK $\dagger$ \chool of Engineering, De Montfort University, The Gateway, Leicester, LE1 9BH, UK
\end{abstract}

\footnotetext{
* Corresponding author, Tel: +44(0)1162577132; Email: mli@dmu.ac.uk
} 


\title{
Understanding the effects of a polymer on the
} surface dissolution of pharmaceutical cocrystals using combined experimental and molecular dynamics simulation approaches

\author{
Preyanthiny Kirubakarant, Ke Wangt, lan Rosbottom, Richard Barrie Michael \\ Cross $^{\dagger \neq}$ and Mingzhong Lit
}

\begin{abstract}
†School of Pharmacy, De Montfort University, The Gateway, Leicester, LE1 9BH, UK ‡Department of Chemical Engineering, Imperial College London, London, SW7 2BX, UK $\dagger \ddagger$ School of Engineering, De Montfort University, The Gateway, Leicester, LE1 9BH, UK
\end{abstract}

\section{For Title of Contents Use Only}

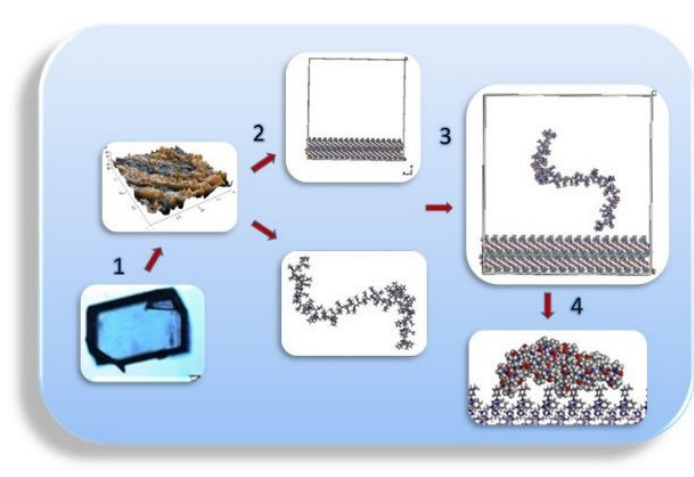

* Corresponding author, Tel: +44(0)1162577132; Email: mli@dmu.ac.uk 


\section{ABSTRACT}

The molecular interactions between the surfaces of cocrystals [i.e., flufenamic acid and theophylline (FFA-TP), flufenamic acid and nicotinamide (FFA-NIC), and carbamazepine and nicotinamide (CBZ-NIC)] and the polymers [i.e., polyethyleneglycol (PEG), polyvinylpyrrolidone (PVP) and copolymer of vinylpyrrolidone $(60 \%) /$ vinyl acetate (40\%) (PVP-VA)] were investigated through combined experimental and molecular dynamics simulation approaches to resolve the mechanisms of cocrystal dissolution and precipitation. It was found that adsorption of the polymers on the surfaces of cocrystals might prevent the precipitation of the parent drug and alter the dissolution rate. The effect of polymers on precipitation could be determined by the cocrystal dissolution rate, the interactions of polymers with the surfaces of cocrystals, the characters of the noncovalent bonds formed between the polymers and the cocrystal surfaces, and the mobility and conformation of the polymers. The etching experiments of single cocrystals revealed that FFA-NIC and CBZ-NIC appeared as surface precipitation cocrystals while FFA-TP could lead to bulk precipitation. Both PVP and PVP-VA were good precipitation inhibitors for FFA-NIC and they could completely inhibit the recrystallization of FFA III on the surfaces of dissolving cocrystals. In addition, as the adsorption of the polymer was slower than dissolution rate of the cocrystals, PVP and PVP-VA could only partially inhibit the recrystallization of CBZ dihydrate on the surface of CBZ-NIC. While PEG had no inhibitory effect on the surface crystallization of FFA-NIC and CBZ-NIC, due to its weak 
interactions with the surfaces of the cocrystals, it enhanced the dissolution performance of FFA-TP. In contrast, PVP and PVP-VA reduced the dissolution rate of FFA-TP and subsequently undermined the performance of cocrystals. Taken together, the approach of combining experimental and molecular dynamics simulation provided insights into the mechanisms of cocrystal dissolution as well as the polymers acting as inhibitory excipients for precipitation/recrystallisation, making contribution to the development of novel formulations.

KEYWORDS: Cocrystals; Precipitation and dissolution inhibitor; Polymer; Flufenamic acid cocrystals; Carbamazepine cocrystals; Molecular dynamics; Molecular modelling. 


\section{INTRODUCTION}

Pharmaceutical cocrystals have been of great interests to the pharmaceutical industries as they provide a wide range of solid forms of APIs (active pharmaceutical ingredient) with modified properties, ${ }^{1}$ and they can improve the bioavailability by increasing the solubility and dissolution rates of poorly water soluble drugs. ${ }^{2}$ However, limitations exist that a stable form of the parent drug tends to recrystallize during dissolution and subsequently lose its improved properties. ${ }^{3}$ Studies on phase transformation and release profiles gave evidence that various polymers could act as precipitation inhibitors during cocrystal dissolution. Meanwhile, it was suggested that interactions of the polymers with the surfaces of cocrystals might be the underlying mechanism. -10 $^{-10}$ Unfortunately, evidence which could thoroughly explain such mechanisms at the molecular level is still largely lacking. ${ }^{4}$

Atomic force microscopy (AFM) is an imaging technique with subnanometer resolution. The etching patterns revealed by AFM have been used to investigate crystal dissolution kinetics and mechanisms, ${ }^{5-9}$ which could be influenced by the interactions of polymers/excipients with the crystals surfaces, the crystal structure itself, and the mutual recognition between the molecules of polymers/excipients and the molecules of crystals. Unfortunately, due to the differing surface chemistry of the crystal facets, one of the downsides of conventional AFM methodology involves the investigations of each individual crystal plane to obtain an overall dissolution behavior. In addition to AFM, molecular dynamics (MD) simulation is recognized as another promising tool to understand interactions, at a molecular level, between the crystal 
surfaces and the polymers/additives. ${ }^{11-19}$ Research with MD simulation indicated that the interaction energies, in particular of hydrogen bonds, between the additives and the crystal faces might play a key role on crystal growth and dissolution. For example, biuret has been used as a shape controlling inhibitor to avoid the formation of needleshaped urea crystals. ${ }^{20} \mathrm{MD}$ simulation suggested that, due to its high affinity and selectivity, biuret could compete with urea for adsorption on the sites of the fast growing face (001) of the lattice, thereby hindered the growth of crystal. However, the same scenario did not seem to apply to the slow growing face (110). Another example of MD simulation suggested polyvinylpyrrolidone (PVP), an additive, as an effective growth inhibitor for the salbutamol sulfate (SS) crystal, ${ }^{16}$ which was in good agreement with the simultaneous experimental study. ${ }^{21} \mathrm{~A}$ recent case was to investigate the effect of binary surfactant-polymer mixtures on crystal surfaces to find out their synergetic effects on drug crystallization and dissolution. 17,22

This research combined the experimental and molecular dynamics simulation approaches mentioned above, aiming to examine the interactions between the surfaces of cocrystals and the polymers. It was expected that the dissolution and precipitation behavior of the pharmaceutical cocrystals could be better interpreted at a molecular level. Meanwhile, the evidence from this research should help with establishing a framework from which the polymeric excipients could be pre-analysed and predicted for their effects on cocrystal dissolution/precipitation before being selected for cocrystal-based formulation development. 
Three pharmaceutical cocrystals, i.e., flufenamic acid and theophylline (FFA-TP), flufenamimic acid and nicotinamide (FFA-NIC), and carbamazepine and nicotinamide (CBZ-NIC), have been chosen as the model drugs for this research. These cocrystals have been extensively studied and characterized, showing that their dissolution behaviors could be regulated by including a polymeric excipient in the dissolution media. 4 , 23 Single cocrystals of FFA-TP, FFA-NIC and CBZ-NIC were firstly synthesized to conduct the etching experiments with or without polyethyleneglycol (PEG), polyvinylpyrrolidone (PVP) or copolymer of vinylpyrrolidone (60\%)/vinyl acetate (40\%) (PVP-VA). The chemical structures of the drugs, coformers and monomer units of the polymers are shown in Table 1. The faces of the cocrystals were indexed based on the morphology prediction of Mercury CSD 4.1.2 (The Cambridge crystallographic Data Centre, Cambridge, UK). The surfaces of the cocrystals were examined further with AFM, Raman spectroscopy and scanning electron microscope (SEM) to investigate the precipitation behavior of the cocrystals. MD simulations were then carried out using commercial software packages of Biovia Materials Studio (V2017 R2). Finally, a cocrystal surface/polymeric excipient composite model was constructed to examine the effects of polymers on the surfaces of cocrystals, involving interaction binding energy, non-covalent bonds (hydrogen bonds, van der Waals forces and electrostatic forces), and mean square displacement (MSD). The MD results were then compared with the experimental observations, aiming to provide a predictive means for the effects of excipients on dissolution and precipitation of pharmaceutical cocrystals. 
Table 1. Chemical structures of cocrystals and monomer units of polymers

\begin{tabular}{|c|c|c|c|c|c|c|}
\hline & $\begin{array}{l}\text { FFA- } \\
\text { TP }\end{array}$ & $\begin{array}{l}\text { FFA- } \\
\text { NIC }\end{array}$ & $\begin{array}{l}\text { CBZ- } \\
\text { NIC }\end{array}$ & PEG & PVP & PVP-VA \\
\hline $\begin{array}{l}\text { Molecular } \\
\text { structure }\end{array}$ & 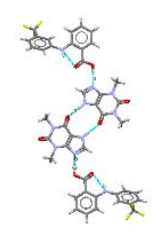 & 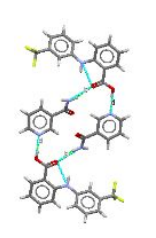 & की & $\mathrm{n}=90$ & $\mathrm{n}=360$ & $\mathrm{n}=303, \mathrm{~m}=202$ \\
\hline $\begin{array}{l}\text { Molar } \\
\text { weight of } \\
\text { material } \\
\text { used in the } \\
\text { experiments }\end{array}$ & 922.78 & 806.70 & 716.78 & 4000 & 40,000 & 51,000 \\
\hline $\begin{array}{l}\text { Number of } \\
\text { monomer } \\
\text { used in the } \\
\text { simulations }\end{array}$ & & & & $N=18$ & $\mathrm{~N}=72$ & $N=31, M=21$ \\
\hline
\end{tabular}

\section{EXPERIMENTAL SECTION}

Materials. Flufenamic acid form I (FFA, $\geq 97 \%$ purity), Nicotinamide (NIC, $\geq 99.5 \%$ purity), Theophylline (TP, $\geq 99.5 \%$ purity) and Carbamazepine III (CBZ, $\geq 99.0 \%$ purity) were purchased from Sigma-Aldrich (Dorset, UK). Poly (ethylene glycol) (PEG) 4000 was purchased from Sigma-Aldrich (Dorset, UK). Plasdone K-29/32 (PVP) and 
Plasdone S-630 copovidone (PVP-VA), which was in a form of 60:40 copolymer of Nvinyl-2-pyrrolidone and vinylacetate, were gifts from Ashland Inc. (Schaffhausen, Switzerland). Acetonitrile (HPLC grade) and Ethyl Acetate (HPLC grade) were purchased from Fisher Scientific UK (Loughborough, UK). Double distilled water was generated from a Bi-distiller (WSC044.MH3.7, Fistreem International Limited, Loughborough, UK) and was used throughout the study.

\section{Experimental Methods. Preparation of single cocrystals.}

Powdered FFA-NIC cocrystals were produced by solvent evaporation. Appropriate amount of FFA and NIC (1:1 molar ratio) were dissolved in cosolvent of acetonitrile $(70 \%)$ and water (30\%). This was then added with FFA-NIC powder, which acted as seeds for the FFA and NIC cosolvent solution. The solution was covered with film containing small holes and was left in a fume cabinet for three days. FFA-NIC single cocrystals were then harvested by filtration.

Single FFA-TP cocrystals were directly synthesised by adding FFA and TP $(1: 1$ molar ratio) in cosolvent of acetonitrile (70\%) and water (30\%). The solution was covered with film containing small holes and was left in a fume cabinet for three days. FFA-TP single cocrystals were then harvested by filtration.

Single CBZ-NIC cocrystals were produced by dissolving CBZ and NIC (1:1 molar ratio) in ethyl acetate. The solution was covered with film containing small holes and was left in a fume cabinet for three days. CBZ-NIC single cocrystals were then harvested by filtration. 
Single cocrystal morphology prediction and face indexing. The morphologies of the cocrystals were created with Mercury CSD 4.1.2 (The Cambridge crystallographic Data Centre, Cambridge, UK) to identify the cocrystal faces that were exposed to the dissolution media during the AFM measurements. The structures of the unit cell of single cocrystals were obtained from Cambridge Structural Database (CSD) (reference codes: ZIQDUA for FFA-TP, EXAQAW for FFA-NIC, and UNEZES for CBZ-NIC). The prediction of the morphologies of single crystals were achieved with the Bravais-Friedel-Donnay-Harker (BFDH) crystal morphology tools. ${ }^{24}$ Validation of the predicted morphologies of the cocrystals were achieved by comparing the predicted PXRD (Powder X-Ray Diffraction) patterns in the Reflex module (BIOVIA Material studio 2017 R2) with the experimental measurements. The index of a crystal face was determined by comparing the observed crystal shape with the simulated morphology.

The indices of single crystal faces were further confirmed by the face-specified PXRD measurements. Each measured single crystal was mounted onto the PXRD sample holder and the measured face was adjusted to be parallel to the disk surface. The face-specified measurements were then compared with the simulated PXRD patterns.

Single cocrystal etching experiment and characterisation. Single cocrystals which had well-defined flat faces were selected under a microscope for the etching dissolution studies. To do that, a single cocrystal was mounted onto a cover glass with double-sided seal tape and, as shown in Table 2, the face of the cocrystal which was of interest was put on the top. The single cocrystal was then immersed in $20 \mathrm{~mL}$ of 
distilled water with or without $0.2 \mathrm{mg} / \mathrm{ml}$ of PEG, PVP or PVP-VA. After a predetermined time intervals, as shown in Table 2, the cocrystal was separated from the solution and carefully patted dry using soft tissue papers. The cocrystal was then air-dried for at least 30 min before undergoing the following investigations.

Firstly, AFM (Park XE100, Park Systems Europe GmbH, Mannheim, Germany) was used to observe the surface which was of interest for the chosen cocrystals. All the measurements were carried out at RT (Room Temperature) in a non-contact mode, using high-aspect-ratio and tapping probe tips (Team Nanotech, Wetzlar, Germany). The results were compared with the relevant AFM images obtained before the etching dissolution tests. The AFM scans were conducted at $10 \times 10 \mu \mathrm{m}^{2}$ for FFATP and FFA-NIC and $5 \times 5 \mu \mathrm{m}^{2}$ for CBZ-NIC, and at least three scans were conducted for any chosen area. The resolution of the measurement was $512 \times 512$ points with equal steps along the $x$ and $y$ directions. Based on the 2D etching patterns, the 3D images of the surfaces were then constructed using XEI software programme (ver. 4.3.0.Build5, Park Systems) and the surface roughness ( $\mathrm{Ra})$ was also obtained. Ra is calculated as:

$$
R a=\left(\frac{1}{L}\right) \int_{0}^{L}|Z(x)| d x
$$

where $L$ is the evaluation length and $Z(x)$ is the profile height function.

EnSpectr R532 ${ }^{\circledR}$ Raman spectroscopy (Enhanced Spectrometry, Inc. Torrance, USA) was also used to observe the surfaces of the single cocrystals following the etching dissolution tests to determine the precipitation behaviour of the parent drug of any single cocrystal. To do that, the single cocrystal was placed in the sample holder and scanned at RT, 20-30mV output power, and a wavelength of $532 \mathrm{~nm}$. The 
integration time was $200 \mathrm{~ms}$, and each spectrum was obtained based on an average of 600 scans.

Finally, SEM micrographs, which were developed with ZEISS EVO HD 15 scanning electron microscope (Carl Zeiss NTS Ltd., Cambridge, UK), was used to determine the surface topology of the single cocrystal after etching dissolution. The single cocrystal was mounted on Agar Scientific G301 0.5" aluminium specimen stub (Agar Scientific Ltd., Stansted, UK) with Agar Scientific G3347N carbon adhesive tab and photographed at a voltage of $10.00 \mathrm{kV}$. The manual sputter coating S150B was used for gold sputtering of the samples. The thickness of gold coating was $15 \mathrm{~nm}$.

Table 2. Time interval for etching dissolution experiments (min)

\begin{tabular}{|l|c|c|c|c|c|}
\hline & $\begin{array}{c}\text { Face } \\
\text { index }\end{array}$ & DW (distilled water) & DW with PEG & $\begin{array}{c}\text { DW with } \\
\text { PVP }\end{array}$ & $\begin{array}{c}\text { DW with } \\
\text { PVP-VA }\end{array}$ \\
\hline FFA-TP & $(0,0,1)$ & 10 & 10 & 10 & 10 \\
\hline FFA-NIC & $(0,-1,1)$ & 7 & 7 & 7 & 7 \\
\hline CBZ-NIC & $(0,0,1)$ & 2 & 2 & 2 & 2 \\
\hline
\end{tabular}

Cocrystal confirmation measurements. Confirmation of the synthesis of single cocrystals was done with Differential Scanning calorimetry (DSC, PerkinElmer Ltd., Beaconsfield, UK), Fourier Transform Infrared Spectroscopy (FTIR) and Powder X-ray diffraction analysis (PXRD).

DSC was used to characterize the melting points. 3-12 $\mathrm{mg}$ of the samples were added to a crimped aluminium pan with a pinhole pierced lid and heated at $20^{\circ} \mathrm{C} / \mathrm{min}$ 
with a nitrogen flow rate of $20 \mathrm{~mL} / \mathrm{min}$. The temperature range was from $50^{\circ} \mathrm{C}$ to $320^{\circ} \mathrm{C}$

FTIR spectra were achieved with an ALPHA interferometer (Bruker UK Limited, Coventry, UK) equipped with a horizontal universal attenuated total reflectance (ART) accessory. 30 scans per spectrum were collected for each sample at a resolution of 2 $\mathrm{cm}^{-1}$. The spectral region was set from 400 to $4000 \mathrm{~cm}^{-1}$ with the OPUS software. All the spectra data were collected at an ambient temperature.

D2 PHASER diffractometer (Bruker U.K. Limited, Coventry, UK) of the PXRD provided the results from $5^{\circ}$ to $35^{\circ}$ at a scanning rate of $0.3^{\circ}(2 \theta) \mathrm{min}^{-1}$. Cu-K $\beta$ was the radiation source and it was used at $30 \mathrm{kV}, 10 \mathrm{~mA}$.

Molecular model and methodology. Dreiding has been widely used in polymer science and engineering, ${ }^{25}$ which could give dynamic calculations in terms of van der Waals, electrostatic and hydrogen bond interactions, and predict the structure of organic, biological and main-group inorganic molecules. ${ }^{26}$ It was selected, in this research, as the force field to model the atomic interactions.

MD simulations were carried out using the Biovia Materials Studio (V2017 R2). The unit cells of FFA-TP, FFA-NIC and CBZ-NIC, which were taken from the CSD, were imported to the Material Studio. The geometries of the unit cells were optimised using the steepest descent algorithm in Forcite module for energy minimization. Details of each of the unit cells after the geometry optimisation were shown in Table S1 in the supporting materials. The surface of the individual crystal with a depth of two unit-cells was then generated by cleaving according to the AFM measurements (Table 2). To integrate the polymers, the crystal surface was extended at both $U$ and $V$ directions at 
different magnitudes (Table 3). A thick vacuum slab of $100 \AA$ was built on top of the crystal surface to form the simulation box. Details of building the crystal surface was shown in Fig. 1.

The numbers of monomers of the polymers used for the etching experiment were shown in Table 1. The property of a polymer and its interaction with the surface of the crystal were greatly affected by its corresponding numbers of monomers, and it would be helpful to build the full polymer chains in simulations. However, the time and resource for computing, as well as the generation of comparable results to the experiments, were to be considered when designing simulations. Therefore, the PEG and PVP built in simulations represented $20 \%$ of the actual polymer weights, i.e.18 and 72 monomer units were allocated for PEG and PVP, respectively. And the chemical structure of the PEG or PVP monomer, as shown in Table 1, was adjusted by extending the head and tail atoms to the required chain length at a torsion angle of $180^{\circ}$. In comparison to PEG or PVP, PVP-VA was more complicated, which the number of monomers had to be reduced to $10 \%$ of its actual polymer weight, i.e. 31 PVP monomers and 21 VA monomers were used for simulations (Table 1). The individual monomer of PVP or VA was then connected randomly as the copolymer PVP-VA using the polymer builder.

Anneal dynamics was used to obtain the global minimum potential energy of a polymer, and it was done by periodically increasing the temperature from $100 \mathrm{~K}$ to $1000 \mathrm{~K}$ followed by a periodic decrease of the temperature from $1000 \mathrm{~K}$ to $100 \mathrm{~K} .10$ repeated annealing cycles were achieved at NVT (constant number of particles, constant volume and constant temperature) ensemble at time step of $1 \mathrm{fs}$. The polymer 
configuration with the lowest energy was selected for MD simulation. The final structure of the polymers and their detailed parameters were shown in Fig. S1 in the supporting materials.

Before starting MD simulations, the polymers were placed approximately $5 \AA$ away from the centre of the crystal surfaces shown in Fig. 1. The initial position of a polymer on crystal surface could vary, whereas the simulation results were not necessarily affected. The polymer-crystal system (Fig. 1) was firstly subjected to geometry optimisation which was achieved, with the steepest descent algorithm, when the energy reached equilibrium. The crystal surface needed to be fixed so that only the polymer was mobile during the simulation. The simulations were performed using periodic boundary conditions at NVT ensemble, and a temperature of $298 \mathrm{~K}$. The cutoff for non-bonded attractions, i.e. van der Waals and electrostatic forces, were $12.5 \AA$, and it was $4.5 \AA$ for hydrogen bonds. The simulations run for 150 ps at a time step of 1 fs until an equilibrium was reached (confirmed by evolution of the energy fluctuation curves where examples were given in Fig. S3). Nosé-Hoover thermostat algorithm was used to control temperature. ${ }^{27}$ The simulations then run for another $50 \mathrm{ps.} \mathrm{The}$ data was saved every 5 ps for each simulation.

Forcite analysis tools were then used to analyse the simulation results, which include non-covalent interaction energies (i.e., hydrogen bond, van der Waals, electrostatic), the binding energy, and mean square displacement (MSD).

The binding energy, $\Delta E_{\text {bind }}$, can accurately reflect the features of the components, e.g. the molecular interaction between the crystal surface and the polymer, which was 
formed from the simulation. Such interaction could be investigated by evaluating the single point total energy of each component in the system: ${ }^{12}$

$$
\Delta E_{\text {bind }}=E_{\text {system }}-\left(E_{\text {polymer }}+E_{\text {surface }}\right)
$$

Where $E_{\text {system }}$ is the total energy of the bonded polymer and crystal surface, $E_{\text {polymer }}$ is the energy of the polymer, and $E_{\text {surface }}$ is the energy of the surface. For this work, the binding energies were calculated at the single point of $200 \mathrm{ps}$, and the more negative of the energy the stronger of the interaction is.

The MSD is defined as a measure of the deviation of the position of a particle with respect to a reference position over time. ${ }^{16} \mathrm{MD}$ simulation demonstrated the mobility of a polymer on the crystal surface, in particular, it helped the determination of whether a polymer is diffusing, transported, or bound on the surface. The MSD is calculated as:

$$
M S D(t)=|r(t)-r(0)|^{2}
$$

where $r(0)$ is the position of the centre of mass of the polymer at the time origin of $150 \mathrm{ps}$, and $r(t)$ is the position of the centre of mass of the polymer at $t$. For this research, the MSD calculation points were from 150ps to $200 p s$.

Table 3. Detailed simulation boxes.

\begin{tabular}{|c|c|c|c|c|c|c|c|}
\hline Drug & $\begin{array}{c}\text { Surface } \\
\text { index }\end{array}$ & \multicolumn{4}{|c|}{$\begin{array}{c}\text { Number of Unit cells for } \\
\text { crystal layer }\end{array}$} & \multicolumn{3}{|c|}{ Dimensions of the simulation } \\
& & $\mathrm{U}$ & $\mathrm{V}$ & $\begin{array}{c}\text { thicknes } \\
\mathrm{b}\end{array}$ & $\mathrm{a}$ & $\mathrm{b}$ & $\mathrm{C}$ \\
\hline & & & & $\mathrm{s}$ & & & \\
\hline FFA-TP & $(0,0,1)$ & 12 & 1 & 2 & 83.9172 & 111.397 & 136.017 \\
& & & 1 & & & & \\
\hline FFA-NIC & $(0,-1,1)$ & 18 & 4 & 2 & 91.8972 & 109.106 & 129.989 \\
\hline
\end{tabular}




\begin{tabular}{|c|c|c|c|c|c|c|c|}
\hline CBZ-NIC & $(0,0,1)$ & 18 & 5 & 2 & 91.7298 & 131.870 & 126.690 \\
\hline
\end{tabular}

Figure 1. Illustration of MD simulation configuration
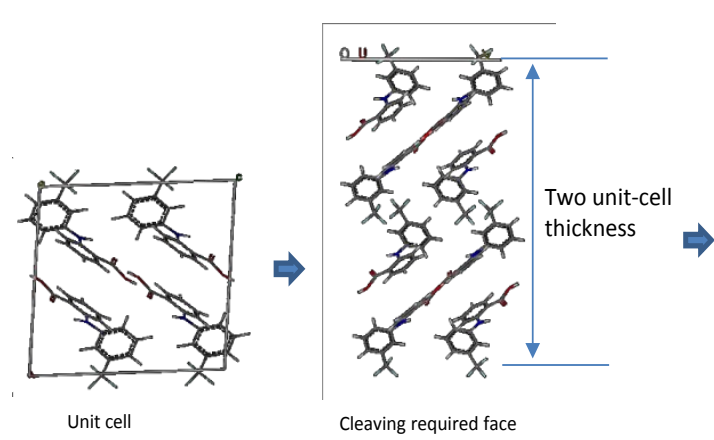

Cleaving required face

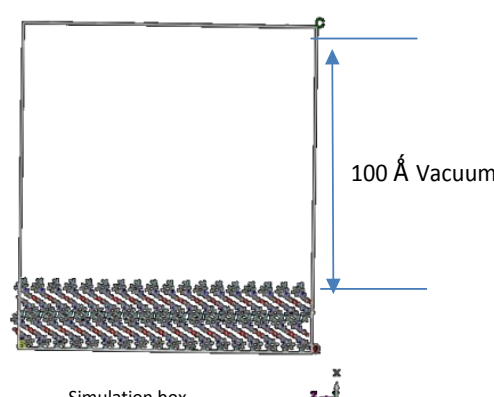

(a) Building crystal surface
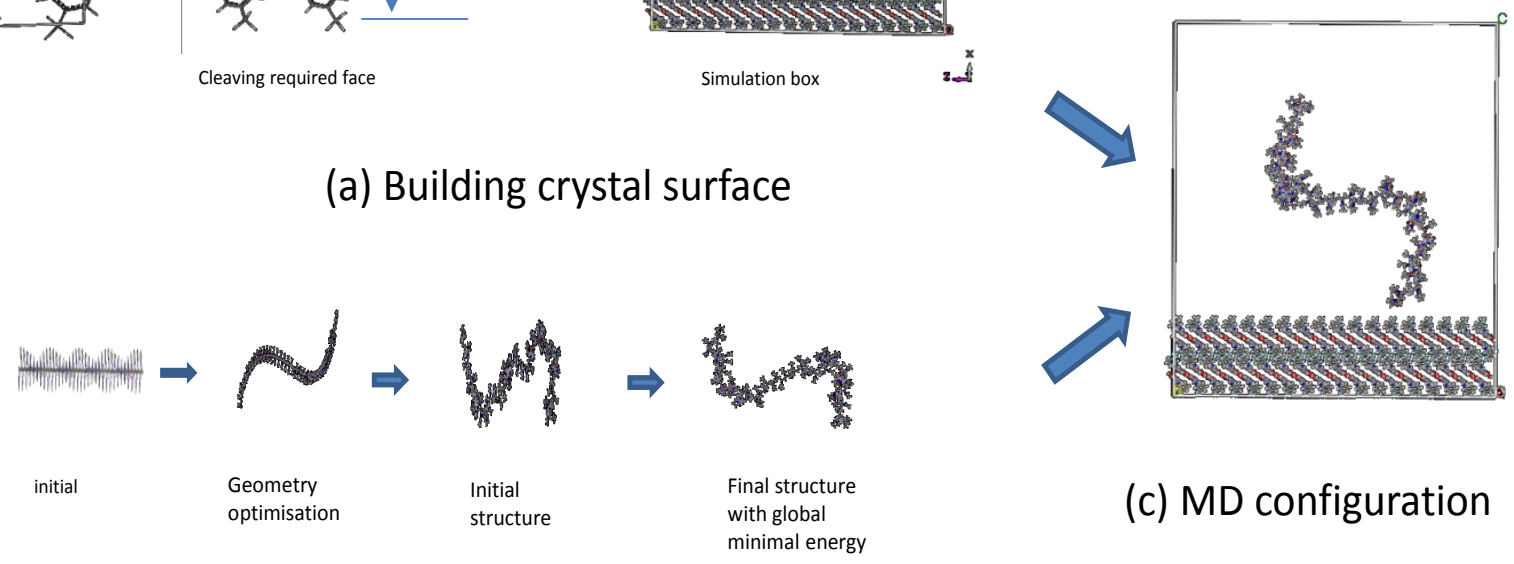

(b) Building polymer

\section{RESULTS}

Morphology prediction and face indices of cocrystals. The identities of the cocrystals of FFA-TP, FFA-NIC and CBZ-NIC were confirmed with PXRD, DSC and FTIR measurements, and they were consistent with previous publications (Fig. S2 in the supporting materials). ${ }^{28,} 29$ 
The morphologies of the cocrystals were determined using Mercury CSD 4.1.2 (Fig. 2), which was consistent with the observed images of the cocrystals. Comparison of the predicted XRD patterns with the experimental PXRD results (Fig. 2) validated the structures of the predicted cocrystals, confirming all of the key characteristic peaks of the cocrystal having been predicted accurately. The predicted morphology of FFATP illustrated dominant big faces of (001) and (00-1), large side faces of (0-10) and (010) and smaller side faces of (100) and (-100). The dominant face of (001) exposed trifluromethylbenzene from the FFA at a perpendicular angle. This suggested that the dominant faces had the least exposed reactive functional groups, whereas the side faces were more reactive due to the existence of more hydrogen-bond donors and acceptors. The predicted morphology of FFA-NIC showed the dominant faces of (011) and (01-1), the smaller faces of (011) and (0-1-1) and the smallest faces of $(-100)$ and (100). The functional group exposed on the dominant face (0-1-1) were carbon, fluorine and hydrogen atoms. The morphology of CBZ-NIC showed the dominant faces of $(011)$ and $(0-1-1)$, and the smaller faces of $(01-1)$ and $(0-11)$, where the face $(011)$ exposed the ring from carbamazepine and nicotinamide which contained the $\mathrm{N}$ atom. The indices of single cocrystal faces were further confirmed by the comparison of the measured face-specified PXRD patterns with the simulations shown in Fig. S4 in the supporting materials.

Using AFM to identify the effects of polymers on cocrystal dissolution. The representative AFM images of the faces of single cocrystals before and after the etching experiments were shown in Fig. 3. Before the etching experiments, the face 
$(0-11)$ of FFA-NIC was relatively flat with its $\mathrm{Ra}$ valued from 0.6 to $2.2 \mu \mathrm{m}$. In comparison, the face (001) of FFA-TP and the face (011) of CBZ-NIC illustrated high roughness from 2.5 to $6.6 \mu \mathrm{m}$ and from 0.8 to $3.5 \mu \mathrm{m}$. After etching in distilled water with or without PEG or PVP-VA, many small interpenetrating rectangle pits along with several long ditches formed on the face (001) of FFA-TP. In contrast, circular pits appeared after etching with PVP. The roughness of the face (001) of FFA-TP increased after dissolution in all solutions, with a maximal increase of $522 \%$ in the presence of PEG and around $350 \%$ in distilled water. PVP caused a small increase of $\mathrm{Ra}$, which was around $115 \%$, and this was in contrast with the moderate increase caused by PVP-VA, which was about $206 \%$.

For the face (0-11) of FFA-NIC, no pit was observed after etching in distilled water or the presence of PEG. However, the roughness of the face significantly increased to $2862 \%$ after etching in distilled water and to $1037 \%$ in the presence of PEG. Small pits appeared on the same face (0-11) after etching in the presence of PVP or PVP-VA, accompanied by a moderate increase of roughness to $300 \%$ in the presence of PVP and to $324 \%$ in the presence of PVP-VA.

No pit was found on the face (001) of CBZ-NIC after etching in distilled water with or without PEG, PVP or PVP-VA. A significant increase of the roughness was observed after etching in distilled water and in the presence of PVP-VA, $1122 \%$ in distilled water and $3960 \%$ in the presence of PVP-VA. A moderate increase of roughness, with a $\mathrm{Ra}$ of $643 \%$, was observed for the same face (001) in the presence of PEG, and this was in contrast with that observed in the presence of PVP, which was $316 \%$. 
Any altered chemical properties of the etched crystal surfaces were determined by Raman spectroscopy (Fig. 4). No change was observed for FFA-TP, indicting no precipitation occurred during etching. This was in consistent with that observed with SEM (Fig. 5), in which no particle was seen on the crystal surfaces.

The characteristic peaks of FFA III appeared in Raman spectra after etching of FFA-NIC single cocrystal in distilled water with or without the presence of PEG. It showed double peaks, one at $370 \mathrm{~cm}^{-1}$ after dissolution of FFA-NIC in distilled water and one at $1170 \mathrm{~cm}^{-1}$ after dissolution in PEG, which was characteristic to FFA III. ${ }^{4}$ These suggested that FFA III might have crystallised on the surfaces of FFA-NIC surfaces during etching. Such suggestion was supported by the SEM images (Fig. 5), demonstrating the presence of rectangular shaped crystals on the dissolving surfaces of FFA-NIC. There was no change observed for the surfaces of FFA-NIC in the presence of PVP or PVP-VA (Fig. 4). This seemed to be consistent with the results obtained by SEM (Fig. 5), which showed pits on the surface of the cocrystal. These results indicated that the recrystallization of the parent drug, FFA, might have been inhibited during cocrystal dissolution as an effect of PVP or PVP-VA.

Recrystallised particles were clearly shown on the surfaces of CBZ-NIC (Fig. 5) after etching with or without PEG, PVP or PVP-VA. While the Raman spectra of the same surfaces (Fig. 4) showed that, in the absence or presence of PEG, the surfaces of CBZ-NIC were exactly the same as those of CBZ dihydrate, from which the characteristic double peaks of $1030 \mathrm{~cm}^{-1}$ and triple peaks between $780 \mathrm{~cm}^{-1}$ to 820 $\mathrm{cm}^{-1}$ were illustrated. ${ }^{30}$ These suggested that the surfaces of CBZ-NIC should have been covered completely by recrystallized CBZ dihydrate during etching. Actually, in the 
presence of PVP or PVP-VA, the Raman spectra of the surfaces of CBZ-NIC surfaces displayed as combined spectra of CBZ-NIC and CBZ dihydrate (Fig.4), indicating that PVP or PVP-VA should be able to partially prevent the recrystallization of $\mathrm{CBZ}$ dihydrate during dissolution. 
Figure 2. Crystal morphology prediction and face index

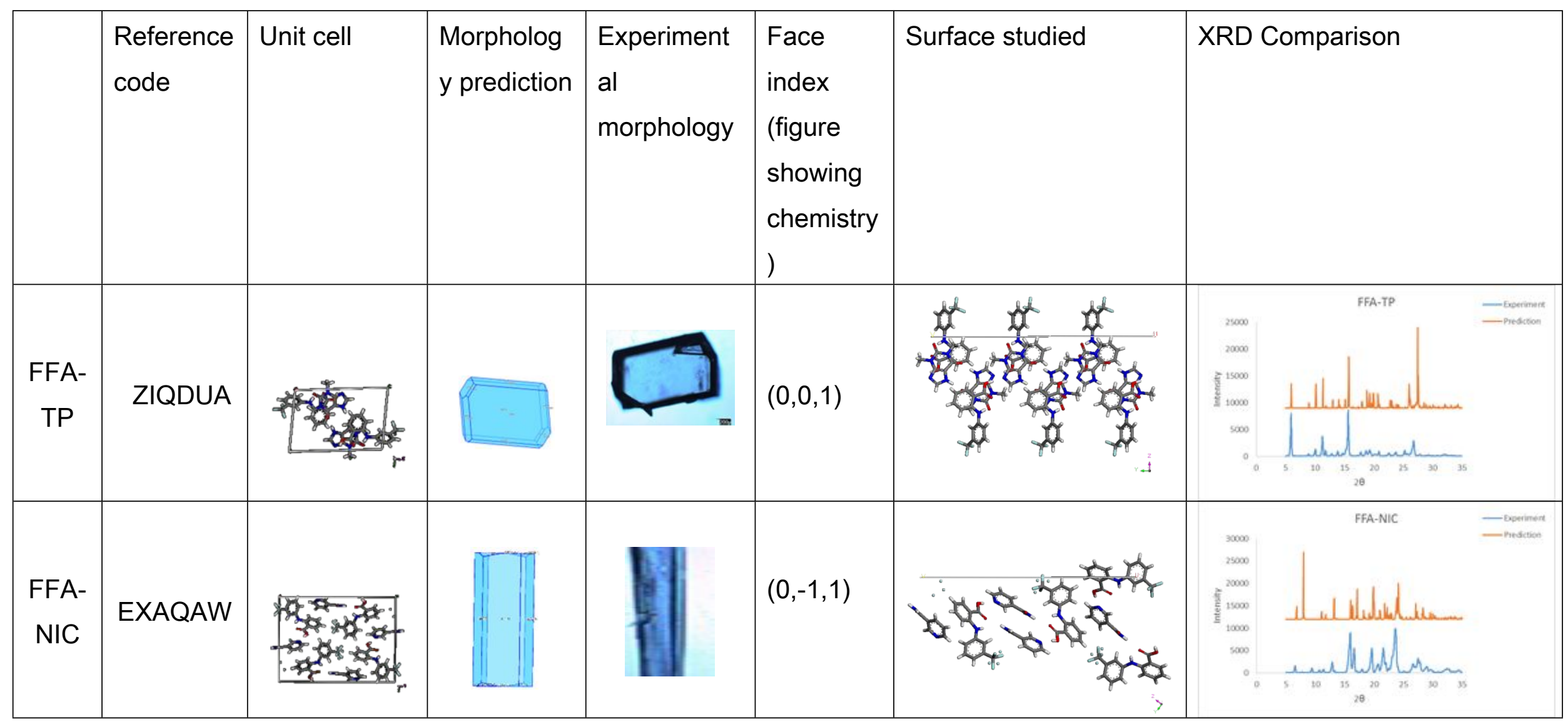




CBZ-


Figure 3. AFM images of cocrystals

\begin{tabular}{|c|c|c|c|c|c|c|c|c|c|}
\hline & Before & After & $\begin{array}{c}\text { 3D and \% } \\
\text { increase }\end{array}$ & Before & After & $\begin{array}{c}3 \mathrm{D} \text { and } \% \\
\text { increase }\end{array}$ & Before & After & $\begin{array}{c}3 \mathrm{D} \text { and } \% \\
\text { increase }\end{array}$ \\
\hline $\begin{array}{l}\text { Distilled } \\
\text { Water }\end{array}$ & & & & & & & & & \\
\hline PEG & & & & & & & & & \\
\hline $\mathrm{Ra}(\mu \mathrm{m})$ & 3.3 & 20.8 & 522 & 2.2 & 24.9 & 1037 & 3.5 & 25.7 & 643 \\
\hline PVP & & & & & & & & & \\
\hline PVP-VA & & & & & & & & & \\
\hline $\mathrm{Ra}(\mu \mathrm{m})$ & 6.6 & 20.1 & 206 & 0.6 & 2.6 & 325 & 0.8 & 34.1 & 3960 \\
\hline
\end{tabular}


Figure 4. Raman spectroscopy results of the cocrystal surfaces before and after etching experiments

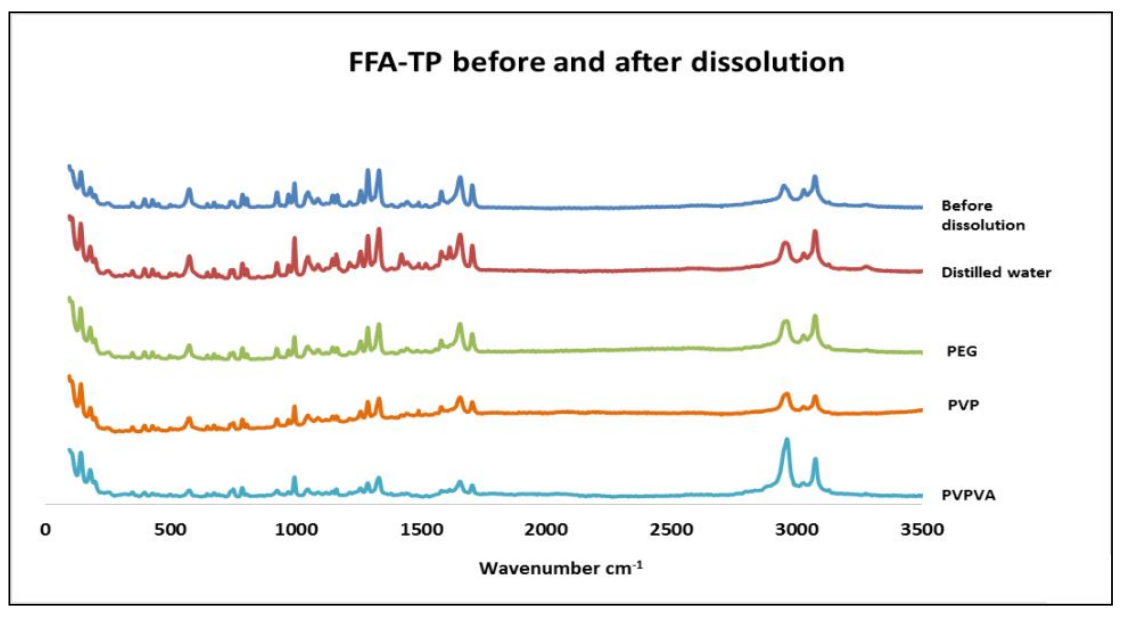

(a) FFA-TP surface

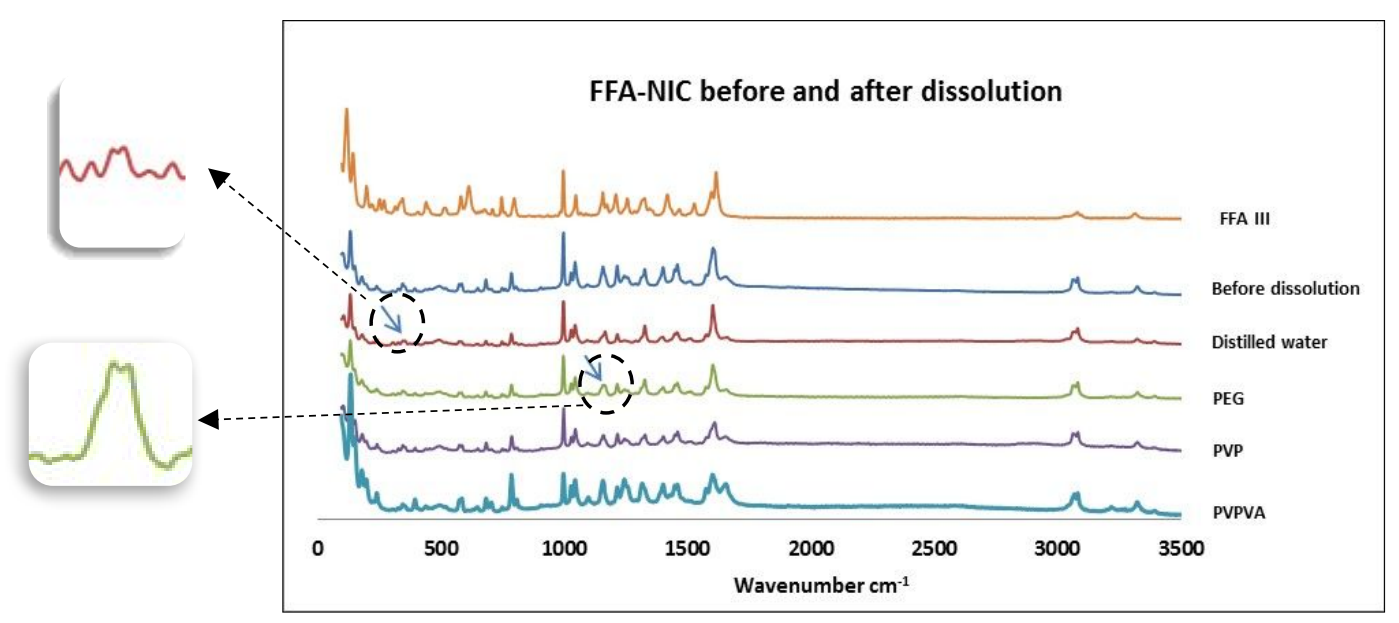

(b) FFA-NIC surface

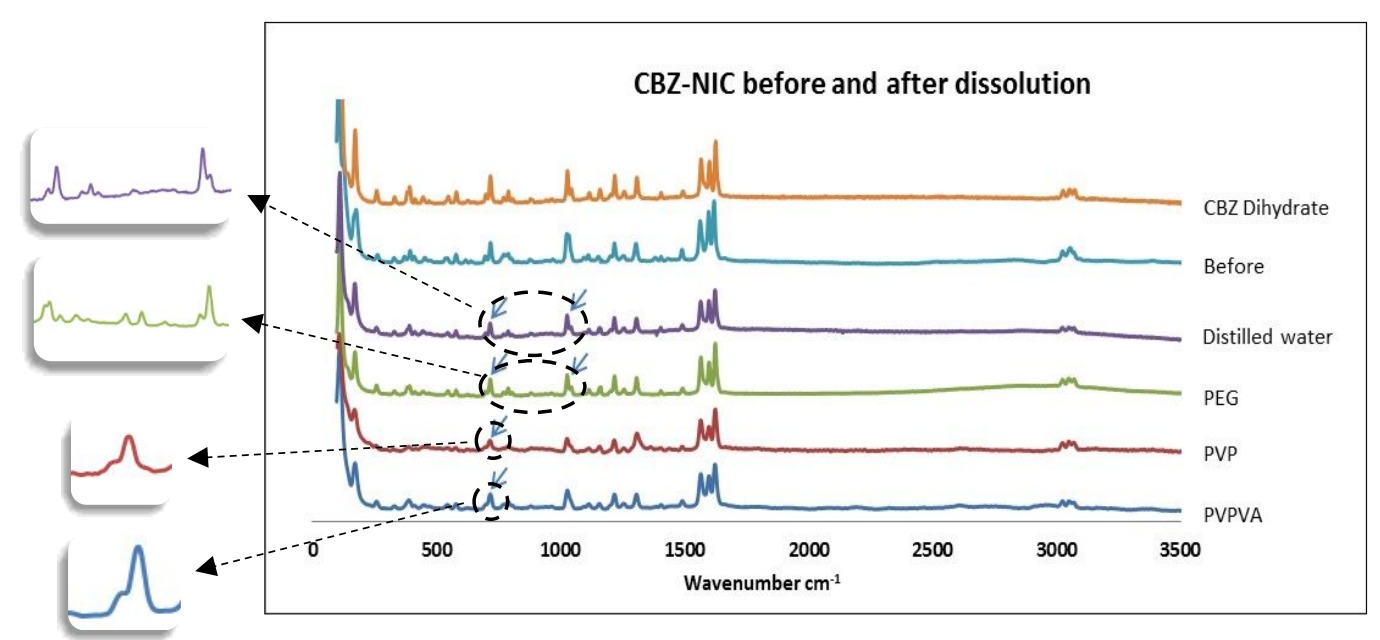


(c) CBZ-NIC surface

Figure 5. SEM results of the cocrystal surfaces before and after etching experiments

\begin{tabular}{|c|c|c|c|}
\hline & FFA-TP & FFA-NIC & CBZ-NIC \\
\hline Before & & & \\
\hline dissolution & & & \\
\hline Distilled water & & & \\
\hline PEG & & & \\
\hline PVP-VA & & & \\
\hline
\end{tabular}

\subsection{Using MD simulation to identify the effect of polymers on cocrystal dissolution}

MD simulations were conducted to determine the change of the dynamic behaviour of polymers on the surfaces of cocrystals. A simple system was chosen by not including any solvent molecules in the work. This was differed from the real system, whereas the simulated results only aimed to serve as qualitative indictors of the inhibitory effects of polymers on parent drug precipitation during dissolution. 
Nevertheless, the MD simulation allowed visualization of the kinetic effects of polymers on the surfaces of cocrystals.

As shown in Fig. 6, the PEG, PVP and PVP-VA could approach and then adsorb on the surface (0-11) of FFA-NIC. The approach of PEG on the surface of FFA-NIC reached equilibrium within 30 ps. After that, there was a steady adsorption of PEG on the crystal surface. Both PVP and PVP-VA were able to approach and interact with the surface of FFA-NIC after 10 ps. PVP started to adsorb on the surface after $60 \mathrm{ps,}$ and the whole PVP chain tended to coil and distribute evenly. Whereas some fragments of PVP-VA chain seemed to be quite far away from the surface, and the PVP-VA chain distributed evenly on the cocrystal surface after $150 \mathrm{ps}$. These indicated that PVP-VA should have the lowest adsorbing rate on the surface in comparison to other polymers. Regarding the face (001) of FFA-TP and CBZ-NIC, PEG had the fastest adsorbing rate and PVP-VA had the lowest one. The snapshots taken at different simulation times were shown in Fig. S5 and Fig. S6 in the supporting materials.

The conformation of polymers on the surfaces of cocrystal at the equilibrium point of 200ps was shown in Fig. 7, showing significant distortion of chain conformation of each of polymers in comparison with the original ones. The binding energies between the polymers and the surfaces of cocrystals were calculated at the same time (Table 4). It can be seen that all of the calculations were negative, implying that the adsorption process of the polymers on the surfaces of cocrystals were exothermic. PEG had the lowest binding energy with each of induvial cocrystal surfaces among three polymers while as PVP had the highest binding energy, which could lead to the most significant 
change of PVP conformation. As shown from the conformations at equilibrium (Fig. 7), PVP did not spread out on the surfaces of the cocrystals. Instead, it coiled up, in particular for the surface (0-11) of FFA-NIC. The detailed intermolecular interaction energies, i.e., hydrogen bonding, van der Waals, electrostatic, between the polymers and surfaces of cocrystals at the equilibrium point of 200ps were also calculated in Table 4. Among the chosen polymers, PEG had the lowest electrostatic and van der Waals energies and it generally laid flat and straight against the surfaces of the cocrystals (Fig. 7). PEG could only form hydrogen bonding with the surface (001) of FFA-TP, as a consequence of the arisen O from TP and OH from PEG (Fig.7), despite the fact that the energy of the hydrogen bond was extremely low. Neither the face (011) of FFA-NIC nor the face (011) of CBZ-NIC formed hydrogen bonds with PEG, due to the lack of hydrogen donor or receptor on the surfaces of the cocrystals (Fig. 2). The van der Waals energies for PVP and PVP-VA were comparable (Table 4), and no hydrogen bonding was observed between PVP or PVP-VA and the surfaces of the cocrystals. The negative electrostatic energy for PVP was strong whereas its van der Waals interaction was weak. The van der Waals energies and electrostatic attractions between PVP-VA and the surfaces of the cocrystals were comparable. And PVP-VA was able to spread out and bind all the surfaces of the cocrystals (Fig. 7).

Fig. 8 demonstrated the mobility of the polymers on the surfaces of the cocrystals. It seemed that PVP-VA had the highest mobility for both of the surface (001) of FFATP and the surface (0-11) of FFA-NIC. PVP had a slightly higher mobility than PEG for surface (0-11) of FFA-NIC. No significant difference was observed for the mobility of PEG, PVP or PVP-VA regarding the surface (011) of CBZ-NIC. 
Table 4. The interaction energies of the polymers and cocrystal surfaces

\begin{tabular}{|l|l|l|l|l|l|}
\hline \multicolumn{2}{|l}{ Crystal and polymer } & $\begin{array}{l}\text { Binding } \\
\text { energy }\end{array}$ & $\begin{array}{l}\text { Hydrogen } \\
\text { bonding }\end{array}$ & $\begin{array}{l}\text { van der } \\
\text { Waals }\end{array}$ & $\begin{array}{l}\text { Electrostati } \\
\text { C }\end{array}$ \\
\hline \multirow{4}{*}{ FFA-TP } & PEG & -44.415 & -0.016 & 14.55 & -12.17 \\
\cline { 2 - 6 } & PVP & -149.05 & 0 & 351.075 & -1734.36 \\
\cline { 2 - 6 } & PVP-VA & -90.48 & 0 & 318.27 & -490.02 \\
\hline \multirow{3}{*}{ FFA-NIC } & PEG & -54.823 & 0 & 7.948 & -10.271 \\
\cline { 2 - 6 } & PVP & -161.68 & 0 & 360.30 & -1740.83 \\
\cline { 2 - 6 } & PVP-VA & -122.90 & 0 & 255.91 & -490.84 \\
\hline \multirow{2}{*}{ CBZ-NIC } & PEG & -45.151 & 0 & 16.185 & -13.816 \\
\cline { 2 - 6 } & PVP & -208.835 & 0 & 350.682 & -1729.835 \\
\cline { 2 - 6 } & PVP-VA & -161.156 & 0 & 216.367 & -483.479 \\
\hline
\end{tabular}


Figure 6. Snapshot of a polymer on FFA-NIC (0-11) surface at $298 \mathrm{~K}$ at different simulation times

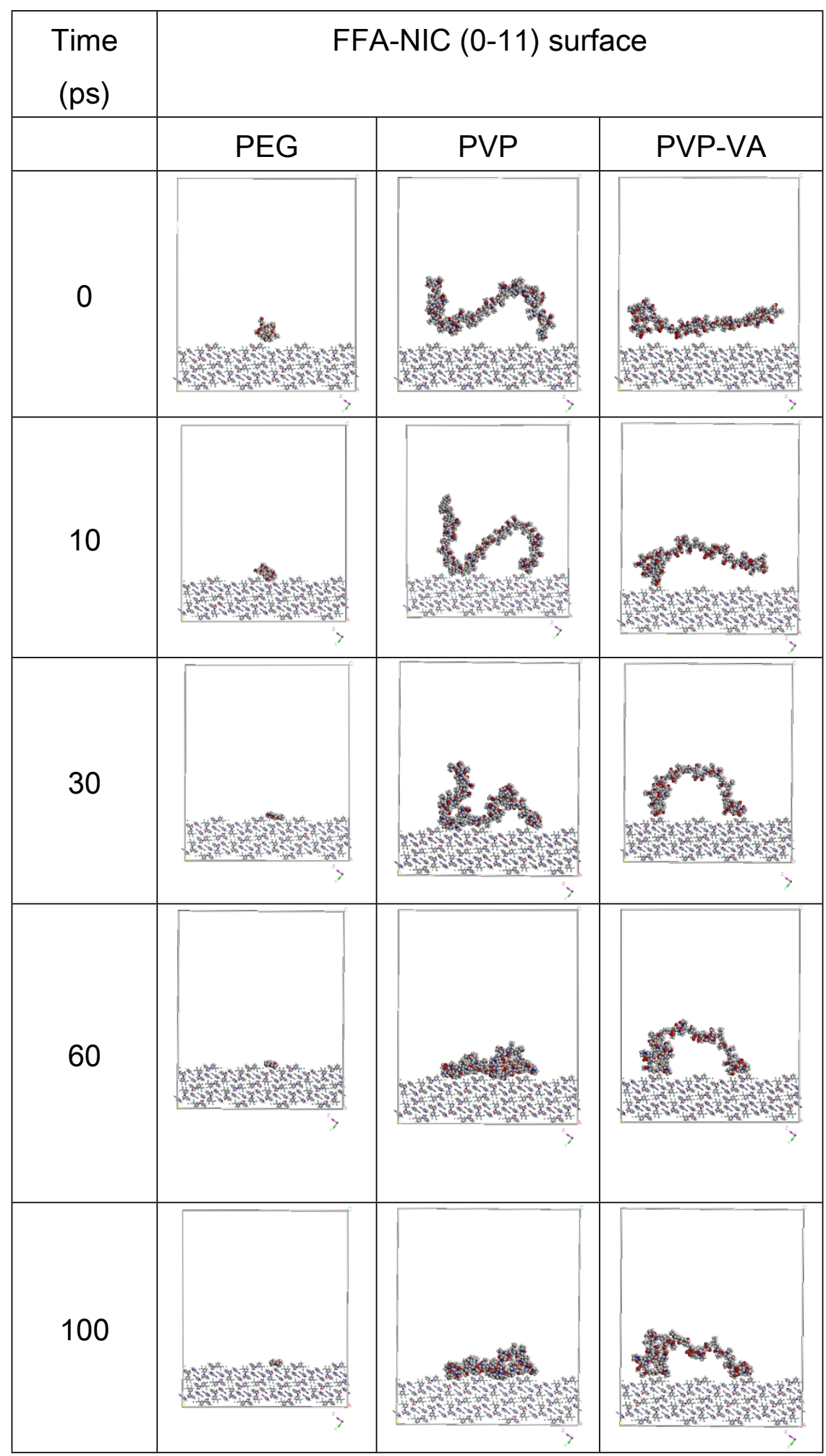




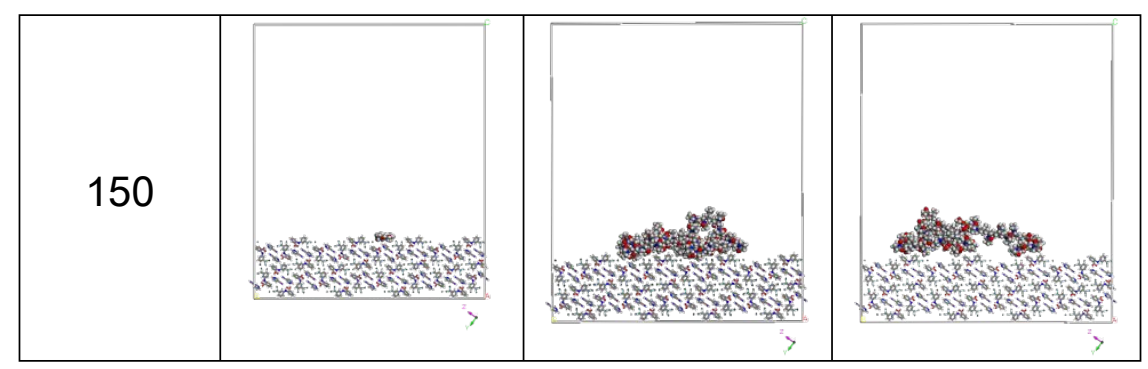


Figure 7. Conformation of polymers on FFA cocrystal surfaces at equilibrium at $298 \mathrm{~K}$ and 200ps.

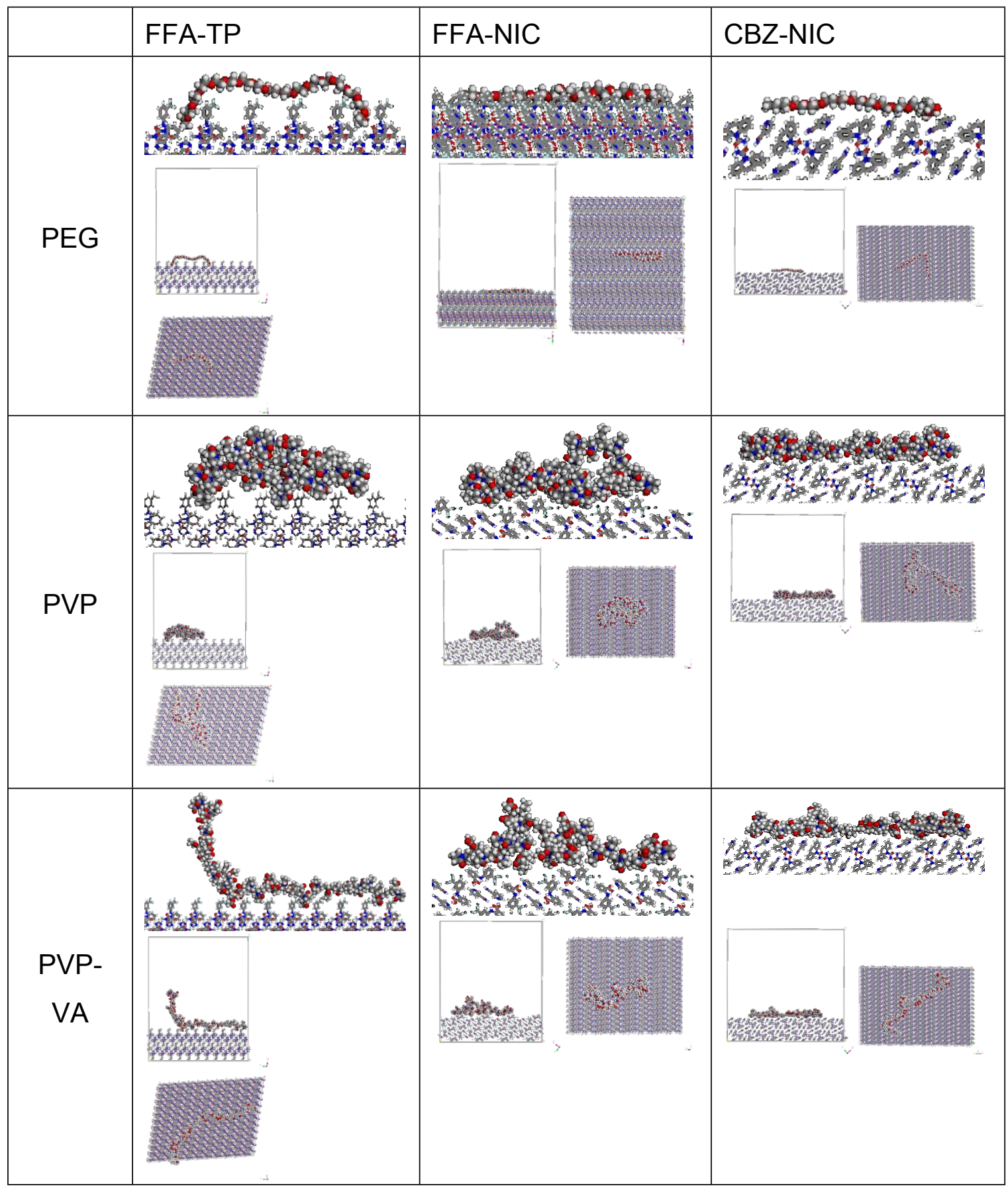


Figure 8. Mean square displacement of a polymer on the crystal surface at equilibrium

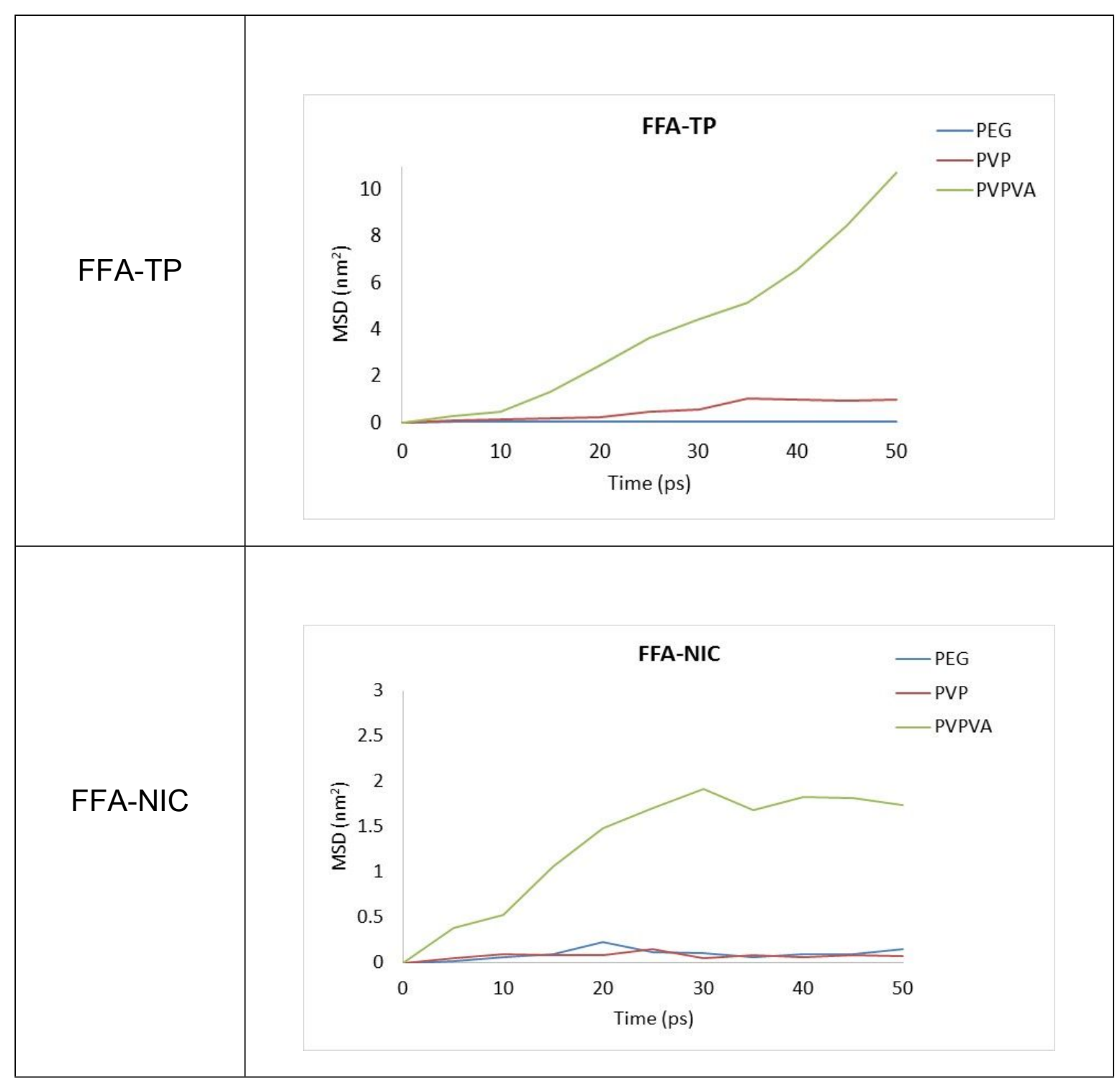




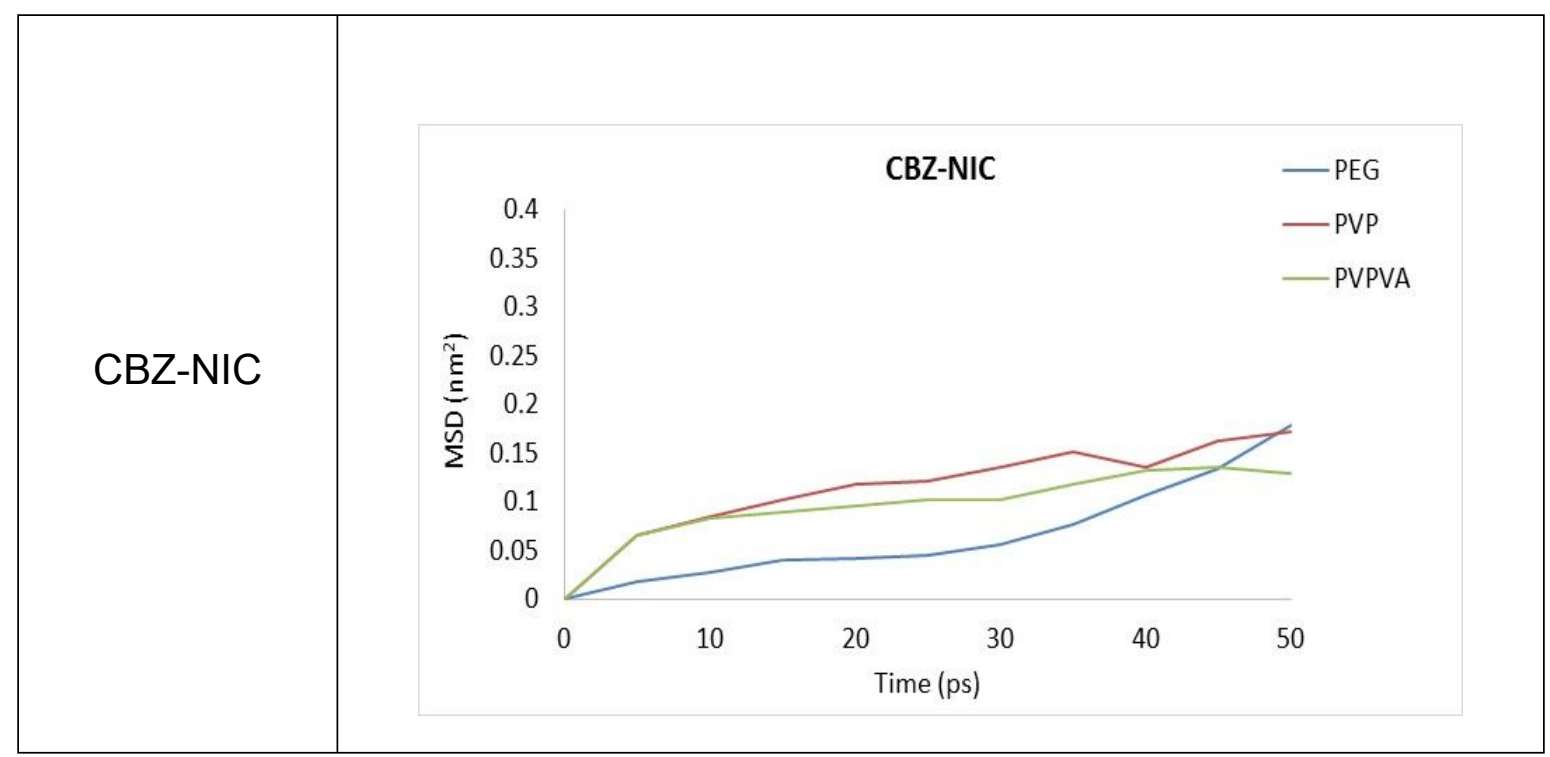

\section{DISCUSSION}

Precipitation of the parent drug could take place on the surface of a cocrystal during dissolution, which was known as surface precipitation mechanism, and it was called bulk precipitation mechanism if it happened in the bulk solution. ${ }^{4}$ Both mechanisms depended on cocrystal dissolution rate and environments, such as $\mathrm{pH}$ and the dissolution medium components. ${ }^{4}$

In this research, Raman spectra and SEM investigations demonstrated that both FFA-NIC and CBZ-NIC undertook surface precipitation when being etched by distilled water, where FFA III crystallised and covered the surface of FFA-NIC, and CBZ dihydrate precipitated on the surface of CBZ-NIC (Fig. 4 and Fig. 5). Therefore, both FFA-NIC and CBZ-NIC belonged to surface precipitation cocrystals. In contrast, FFA- 
TP belonged to bulk precipitation cocrystals as there was no parent drug precipitation after its etching in distilled water. On the other hand, the chemical properties of the surface of FFA-TP remained the same after etching (Fig. 4) and the pits illustrated by SEM (Fig. 5) should be caused by the detachment of FFA and TP molecules from the dissolving surface.

There was evidence that the cocrystal based formulations could significantly improve the dissolution and solubility of the poorly water soluble drugs. However, those improved properties could be undermined if the parent drug precipitated directly onto the dissolving surface of the cocrystal and acted as a coating layer. Previous evidence showed that FFA-NIC and its parent drug, FFA, had similar DPPs (dissolution performance parameter) in dissolution media without inhibitors. ${ }^{4}$ It was also indicated that the CBZ-NIC contained in a 100mg of HPMC matrix tablet had a slower release profile in comparison to the CBZ III contained in the same matrix tablet. ${ }^{23}$ The etching experiments carried out in this research clearly suggested that both PVP and PVP-VA were good surface precipitation inhibitors for FFA-NIC, as they could completely inhibit the recrystallization of FFA III on the dissolving surface of FFANIC (Fig. 4 and Fig. 5). Meanwhile, the presence of PVP or PVP-VA resulted in the combination of the characteristic Raman peaks of CBZ-NIC and CBZ dihydrate (Fig. 4), indicating that the surface of CBZ-NIC had been partially covered by CBZ dihydrate crystals. Subsequently, PVP and PVP-VA should act as partial inhibitors of the recrystallization of CBZ dihydrate on the surface of CBZ-NIC. In contrast, PEG acted as a surface crystallization inhibitor for neither FFA-NIC nor CBZ-NIC (Fig. 3, Fig. 4 and Fig. 5). 
The interactions of the polymers with the surfaces of the cocrystals played essential roles in controlling the dissolution and morphological change of the cocrystals during dissolution. The MD simulations as well as the etching experiment indicated that, in comparison to PVP or PVP-VA, the lowest binding and interaction energies existed between PEG and the surfaces of the cocrystals, in other words, PEG was not an effective inhibitor. In contrast to PVP-VA, PVP had greater binding and interaction energies (Table 4). One thing to be noted was that the calculated interaction energy for PVP was based on $20 \%$ of its actual polymer weight, and that of PVP-VA was based on $10 \%$ of its actual polymer weight. Therefore, it was expected the PVP-VA was a better surface inhibitor because a higher molar weight polymer of PVP-VA had stronger interaction with the surfaces of FFA-NIC or CBZ-NIC in comparison to PVP. Intriguingly, the above theory couldn't be applied to the observations from etching experiments, showing that PVP was more effective in inhibiting parent drug precipitation in comparison to PVP-VA. Additionally smaller increase of Ra was detected for PVP in contrast to PVP-VA (Fig. 3). This observation was consistent with previous DPP investigations for FFA-NIC, demonstrating a 64\% increase of AUC (area under curve) in the presence of PVP whereas a $60 \%$ increase in the presence of PVP-VA. ${ }^{4}$ All of these indicated that other factors, such as noncovalent bonds existed between the polymers and the surfaces of cocrystals or the polymer mobility and conformation, might play important roles in modulating the cocrystal dissolution and parent drug precipitation.

The simulations and etching experiments carried out for this research looked into the biggest and most stable faces of the cocrystals, which were generally from the 
hydrophilic regions of the molecules. These investigations showed that PVP had weak van der Waals interaction and greater electrostatic energy (Table 4), contributing to its low mobility on the surfaces of cocrystals (Fig. 8). Adsorption of PVP on the surfaces of cocrystals led to the formation of a steric hindrance layer. This layer prevented solvent molecules from contacting the surface cocrystal particles and subsequently reduced the dissolution rate. In the meantime, the layer prevented the supersaturated state of the parent drug around the dissolution surface and inhibited the precipitation of the parent drug. Apart from that, a conformational change was observed for PVP when it exhibited the inhibitory effect - PVP molecules coiled up when they adsorbed on the surfaces of the cocrystals. This was in contrast to PVP-VA, which became more mobile on the surfaces of the cocrystals (Fig. 8), rending it difficult to form a stable steric hindrance layer. Therefore, PVP-VA was a less effective precipitation inhibitor for FFA-NIC and CBZ-NIC.

Neither PVP or PVP-VA completely prevented the precipitation of CBZ dihydrate on the surfaces of CBZ-NIC. In comparison to the parent drug (CBZ dihydrate), CBZNIC had a much higher solubility (150 times more), ${ }^{31,32}$ which greatly enhanced its intrinsic dissolution rate. It was likely that the adsorption rate of PVP or PVP-VA was much slower than the dissolution rate of the cocrystals, contributing to recrystallization of CBZ dihydrate on the dissolving surface.

As previously mentioned, there was no parent drug recrystallization during the dissolution of FFA-TP. In addition, adsorption of the polymer of PVP or PVP-VA on the surface of FFA-TP reduced the dissolution rate of the cocrystals. This was in agreement with what was observed in the etching experiments, in which there was a 
slightly increased Ra of FFA-TP due to the presence of PVP or PVP-VA (Fig. 3). Moreover, PVP seemed to be much more effective than PVP-VA as a surface precipitation inhibitor, as a $115 \%$ increase of FFA-TP surface roughness was observed in the presence of PVP in comparison to a $206 \%$ increase in the presence of PVP-VA (Fig. 3). PEG couldn't be considered here as a precipitation inhibitor, instead, it behaved more as a dissolution/solubility enhancer for FFA-TP.

Inclusion of a precipitation inhibitor in bulk precipitation cocrystals, e.g. FFA-TP, could potentially undermine its improved solubility and dissolution rate. For example, previous research showed that PVP-VA reduced the DPP measured for FFA-TP in the presence of PVP-VA. ${ }^{4}$ The presence of PEG rendered an increased Ra of FFA-TP during etching in contrast to the Ra measured in distilled water (Fig. 3). This complied with what was published previously that an significantly increased DPP was observed for FFA-TP in the presence of PEG. ${ }^{4}$

\section{CONCLUSIONS}

This research used combined experimental and molecular dynamics simulation approaches to examine the molecular interactions between the surfaces of cocrystals (FFA-TP, FFA-NIC and CBZ-NIC) and the polymers (PEG, PVP and PVP-VA) during dissolution, aiming to reveal the dissolution and precipitation mechanisms of cocrystals. It was found that adsorption of the polymers on the surfaces of cocrystals might prevent the precipitation of parent drug and altered the dissolution rate. Many factors could affect the inhibitory effect of the polymers, including the cocrystal 
dissolution mechanism, interactions between the cocrystal surfaces and the polymers, and the mobility and conformation of the polymers.

The etching experiments carried out in this research demonstrated FFA-NIC and CBZ-NIC as surface precipitation cocrystals, and FFA-TP as bulk precipitation cocrystals. Both PVP and PVP-VA showed their characters as surface precipitation inhibitors for FFA-NIC, and they could completely inhibit the recrystallization of FFA III on the surface of FFA-NIC. In contrast, PVP and PVP-VA could only partially inhibit the recrystallization of CBZ dihydrate on the surface of CBZ-NIC, which should be attributed to a slower adsorption rate of the polymers than the dissolution rate of the cocrystals. PEG was not considered as surface crystallization inhibitor for FFA-NIC and CBZ-NIC due to its weak interactions with the surfaces of the cocrystals. The presence of PVP or PVP-VA reduced the dissolution rate of FFA-TP, undermining the solubility and dissolution of the cocrystals. Therefore, for bulk precipitation cocrystals, an addition of a solubiliser, such as PEG, to the formulation should greatly enhance the efficiency of dissolution.

Taken together, this research provided insights into the mechanisms of cocrystal dissolution and how the polymeric excipients exhibited their inhibitory functions for precipitation, which were of great value to the development of novel cocrystal-based formulations. It was highly promising that such approaches would be set up as an essential tool during the routine process of manufacturing pharmaceutical cocrystals. Finally, it is worth noting that the MD simulations without considering the dissolution medium may be too simplistic as a tool for the selection of polymeric excipients in the formulation because nucleation and growth of the parent drug could take place in the 
diffusion layer. Therefore, a full MD simulation will be required to provide a comprehensive understanding of the dissolution and recrystallisation mechanisms of pharmaceutical cocrystals.

\section{ASSOCIATED CONTENT}

\section{Supporting information}

The Supporting Information is available free of charge available online

Table S1: The unit cell parameters.

Figure S1: the initial structures of polymers used in the simulations

Figure S2: cocrystal confirmation: (a) FFA-TP characterisation; (b) (b) FFA-NIC characterisation; (c) Characterisation of CBZ-NIC;

Figure S3: Evolution of energy profiles of FFA-NIC with PEG.

Figure S4: Comparison of the measured PXRD patterns of the indexed surfaces of the single cocrystals with simulations.

Figure S5: Snapshots of polymers on FFA-TP surfaces at 298k at 0, 10, 30, 60, 100 and 150ps.

Figure S6: Snapshots of polymers on CBZ-NIC surfaces at 298k at 0, 10, 30, 60, 100 and 150ps. 


\section{AUTHOR INFORMATION}

\section{Corresponding Author}

*Tel: +44-1162577132; E-mail:mli@dmu.ac.uk

ORCID

Mingzhong LI: 0000-0002-9532-9049

\section{Notes}

The authors declare no competing financial interest.

\section{ACKNOWLEDGEMENT}
We would like to thank Dr. Rachel Armitage and Leonie Hough for taking the SEM images of the crystals.

\section{REFERENCE}

1. Qiao, N., et al., Pharmaceutical cocrystals: An overview. International Journal of Pharmaceutics, 2011. 419(1-2): p. 1-11.

2. Shan, N., et al., Impact of pharmaceutical cocrystals: the effects on drug pharmacokinetics. Expert Opinion on Drug Metabolism \& Toxicology, 2014. 10(9): p. 1255-1271.

3. Greco, K. and R. Bogner, Solution-Mediated Phase Transformation: Significance During Dissolution and Implications for Bioavailability. Journal of Pharmaceutical Sciences, 2012. 101(9): p. 2996-3018. 
4. Guo, M., et al., Insight into Flufenamic Acid Cocrystal Dissolution in the Presence of a Polymer in Solution: from Single Crystal to Powder Dissolution. Molecular Pharmaceutics, 2017. 14(12): p. 4583-4596.

5. Wen, H., et al., How Solvents Affect Acetaminophen Etching Pattern Formation: Interaction between Solvent and Acetaminophen at the Solid/Liquid Interface. The Journal of Physical Chemistry B, 2004. 108(7): p. 2270-2278.

6. Wen, H., K.R. Morris, and K. Park, Synergic Effects of Polymeric Additives on Dissolution and Crystallization of Acetaminophen. Pharmaceutical Research, 2008. 25(2): p. 349-358.

7. Danesh, A., et al., An In Situ Dissolution Study of Aspirin Crystal Planes (100) and (001) by Atomic Force Microscopy. Pharmaceutical Research, 2001. 18(3): p. 299-303.

8. Wen, H., K.R. Morris, and K. Park, Hydrogen bonding interactions between adsorbed polymer molecules and crystal surface of acetaminophen. Journal of Colloid and Interface Science, 2005. 290(2): p. 325-335.

9. Wen, H., K.R. Morris, and K. Park, Study on the Interactions Between Polyvinylpyrrolidone (PVP) and Acetaminophen Crystals: Partial Dissolution Pattern Change. Journal of Pharmaceutical Sciences, 2005. 94(10): p. 21662174.

10. Gajda, M., et al., The role of the polymer matrix in solvent-free hot melt extrusion continuous process for mechanochemical synthesis of pharmaceutical cocrystal. European Journal of Pharmaceutics and Biopharmaceutics, 2018. 131: p. 48-59.

11. Wang, C., et al., Effects of solvent and supersaturation on crystal morphology of cefaclor dihydrate: a combined experimental and computer simulation study. CrystEngComm, 2016. 18(47): p. 9085-9094.

12. Zeng, J.-P., et al., Molecular dynamics simulation on the interaction between polymer inhibitors and anhydrite surface. Surface and Interface Analysis, 2015. 47(9): p. 896-902.

13. Anwar, J., et al., Mode of Action and Design Rules for Additives That Modulate Crystal Nucleation. Angewandte Chemie International Edition, 2009. 48(9): p. 1596-1600. 
14. Hadicke, E., et al., Molecular dynamics simulations of the incrustation inhibition by polymeric additives. Physical Chemistry Chemical Physics, 1999. 1(17): p. 3891-3898.

15. Salvalaglio, M., et al., Uncovering Molecular Details of Urea Crystal Growth in the Presence of Additives. Journal of the American Chemical Society, 2012. 134(41): p. 17221-17233.

16. Yani, Y., P.S. Chow, and R.B.H. Tan, Molecular Simulation Study of the Effect of Various Additives on Salbutamol Sulfate Crystal Habit. Molecular Pharmaceutics, 2011. 8(5): p. 1910-1918.

17. Zhu, W., et al., Atomistic simulations of aqueous griseofulvin crystals in the presence of individual and multiple additives. Chemical Engineering Science, 2012. 73: p. 218-230.

18. Ouyang, D., Investigating the molecular structures of solid dispersions by the simulated annealing method. Chemical Physics Letters, 2012. 554: p. 177184.

19. Chan, T. and D. Ouyang, Investigating the molecular dissolution process of binary solid dispersions by molecular dynamics simulations. Asian Journal of Pharmaceutical Sciences, 2018. 13(3): p. 248-254.

20. Salvalaglio, M., et al., Controlling and Predicting Crystal Shapes: The Case of Urea. Angewandte Chemie International Edition, 2013. 52(50): p. 1336913372.

21. Xie, S., et al., Direct Precipitation of Micron-Size Salbutamol Sulfate: New Insights into the Action of Surfactants and Polymeric Additives. Crystal Growth \& Design, 2010. 10(8): p. 3363-3371.

22. Zhu, W., et al., Atomistic simulation study of surfactant and polymer interactions on the surface of a fenofibrate crystal. European Journal of Pharmaceutical Sciences, 2011. 42(5): p. 452-461.

23. Li, M., et al., Investigation of the Effect of Hydroxypropyl Methylcellulose on the Phase Transformation and Release Profiles of CarbamazepineNicotinamide Cocrystal. Pharmaceutical Research, 2014. 31(9): p. 23122325.

24. Docherty, R., et al., Application of Bravais-Friedel-Donnay-Harker, attachment energy and Ising models to predicting and understanding the morphology of 
molecular crystals. Journal of Physics D: Applied Physics, 1991. 24(2): p. 8999.

25. Mayo, S.L., B.D. Olafson, and W.A. Goddard, DREIDING: a generic force field for molecular simulations. The Journal of Physical Chemistry, 1990. 94(26): p. 8897-8909.

26. Gartner, T.E. and A. Jayaraman, Modeling and Simulations of Polymers: A Roadmap. Macromolecules, 2019. 52(3): p. 755-786.

27. Evans, D.J. and B.L. Holian, The Nose-Hoover thermostat. The Journal of Chemical Physics, 1985. 83(8): p. 4069-4074.

28. Fábián, L., et al., Cocrystals of Fenamic Acids with Nicotinamide. Crystal Growth \& Design, 2011. 11(8): p. 3522-3528.

29. Aitipamula, S., et al., Cocrystallization with flufenamic acid: comparison of physicochemical properties of two pharmaceutical cocrystals.

CrystEngComm, 2014. 16(26): p. 5793-5801.

30. Qu, S., et al., Role of polymers in solution and tablet-based carbamazepine cocrystal formulations. CrystEngComm, 2016. 18(15): p. 2664-2678.

31. Good, D.J. and N. Rodríguez-Hornedo, Solubility Advantage of Pharmaceutical Cocrystals. Crystal Growth \& Design, 2009. 9(5): p. 22522264.

32. Li, M., N. Qiao, and K. Wang, Influence of Sodium Lauryl Sulfate and Tween 80 on Carbamazepine-Nicotinamide Cocrystal Solubility and Dissolution Behaviour. Pharmaceutics, 2013. 5(4): p. 508. 